\title{
The Effect of Virtual Rehabilitation Added to an Accelerated Rehabilitation Program After Anterior Cruciate Ligament Reconstruction: A Randomized Controlled Trial
}

\author{
Zehra Betul Karakoc (D), Tugba Kuru Colak (D), Zubeyir Sari (D), Mine Gulden Polat (D) \\ Marmara University, Faculty of Health Sciences, Department of Physiotherapy and Rehabilitation, Istanbul, Turkey
}

Correspondence Author: Zehra Betul Karakoc

E-mail: fztbetulmarmara@gmail.com

Received: $18.01 .2018 \quad$ Accepted: 04.04.2018

\begin{abstract}
Objective: This study aimed to determine the effectiveness of virtual rehabilitation on balance and functionality in patients with anterior cruciate ligament (ACL) reconstruction.

Methods: A total of 22 males who had undergone ACL reconstruction were divided into Nintendo and control groups. Both of the groups received six week accelerated rehabilitation in our department, and the Nintendo WiiC (Nintendo, Washington, USA) balance games were added to Nintendo group after three weeks for forty minutes a day, three times a week. Patients were evaluated for pain (visual analog scale), functionality (Lower Extremity Functional Scale), center of gravity (COG) and balance (Nintendo WiiC) at the baseline and end of the 3th and 6th week of rehabilitation program.

Results: Similar improvements were determined in the pain, functionality, COG and balance scores of the two groups at the end of the treatment program and there were no significant differences between the groups in the amount of change in all parameters $(p=0.256,0.393,0.707,1.000)$. Conclusion: According to the results of this study the Nintendo Wii $\bigcirc$ balance games applied in the clinic under physiotherapist supervision did not change the outcome of the rehabilitation in early period after $\mathrm{ACL}$ reconstruction.
\end{abstract}

Keywords: Anterior Cruciate Ligament, Nintendo Wii@, Postural Balance.

\section{INTRODUCTION}

Anterior cruciate ligament $(A C L)$ is the most injured and surgically reconstructed ligament in the body (1). While tibiofemoral joint biomechanics are impaired after injury (2), damage also occurs in the mechanoreceptors in the $\mathrm{ACL}$ insertion zone (3). The change in proprioception affects many factors such as level of physical activity (4), balance (5), restoration of muscle strength of the quadriceps $(\mathrm{Q})$ and reinjury.

The primary goals of $A C L$ reconstruction surgery are to restore knee stabilization and high-level functional activity and correct abnormal movement patterns (6). Although the mechanical stability is obtained with surgery, functional recovery can not be fully achieved when there is continuing damage of the mechanoreceptors (7). The post-operative rehabilitation program after $A C L$ reconstruction is an important factor that reveals the efficacy of the surgical treatment as in many other areas (8). There is no consensus on the ideal treatment algorithm for patients undergoing $\mathrm{ACL}$ reconstruction as there are various clinical practices for the management of this injury (9). Better outcomes may result from reconstruction or improvements to the neuromuscular control of the knee in terms of an early return to daily functional activities and reduced rates of recurrence of the injury (10).

The Nintendo Wii ${ }^{\odot}$ Balance Board (WBB) (Nintendo, Kyoto, Japan), is a component of the popular video game Wii Fit, which was designed as a video game controller, is primarily used together with a video game console and the relevant software. As this system can provide instant feedback and motivation through increased levels of difficulty (11), it has already been integrated into neurological rehabilitation programs for subjects with impaired balance (12).

With developments in technology in recent years, the exercises in the rehabilitation programs have become more enjoyable and interesting with virtual game systems (13). Few studies investigated the efficiency of the virtual games widely used in the rehabilitation after ACL reconstruction surgery. The aim of this study was to investigate the effectiveness of Nintendo $\mathrm{Wii}^{\circ}$ balance games added to the accelerated rehabilitation program after $\mathrm{ACL}$ reconstruction in order to provide new information to the literature in this area. 


\section{METHODS}

This prospective study enrolled patients with MRI proven $A C L$ injury who underwent arthroscopic $A C L$ reconstruction between 26 October 2015 and 26 June 2016 in the Kartal Dr. Lütfi Kırdar Training and Research Hospital. Approval for the study was granted by the Ethics Committee of Marmara University, Health Sciences Institute (26.10.2015 - 4).

The inclusion criteria were as follows: arthroscopic $\mathrm{ACL}$ reconstruction in the previous six months, age $>18$ years, and no other treatment which might affect rehabilitation outcomes. Patients with contraindications for exercise practice, prior lower extremity operations, knee instability, meniscus and posterior cruciate ligament ( $P C L)$ rupture, additional disease or medication that may affect postural control, spinal and lower extremity injuries that can affect physical activity, or congenital deformity, were excluded.

Patients who had signed the informed consent form were divided into two groups using the "Research Randomiser" program (14). Each patient selected a number in a closed envelope, which was sorted for the randomisation process, and the patients were separated into the following two groups; the Nintendo group and the control group. The acclerated rehabilitation program including a modified Shelbourne protocol (15) was applied to both groups by a physiotherapist in our department. The rehabilitation program was divided into four phases. Phase 1 included edema and pain control, and increasing range of motion; Phase 2, weight-bearing and strengthening exercises (thera-band ${ }^{\circledR}$ exercises); Phase 3 and 4 strengthening exercises progressed and functional and balance exercises (with rocker board). The sessions were individual and lasted for 45 minutes, 3 times a week for 6 weeks. Nintendo $\mathrm{Wii}^{\odot}$ balance games were added to the rehabilitation program of the Nintendo group in the 4th week. These games lasted 40 minutes after the main rehabilitation program, and the patients were given a 10 minute rest period before starting the Nintendo $\mathrm{Wii}^{\odot}$ balance games. Each of the soccerheading, skiing, tabletilt and penguinslide games placed in the Nintendo $\mathrm{Wii}^{\odot}$ game console were played for 10 minutes. In the last week of the virtual rehabilitation program, the difficulty levels of the games were increased.

Assessments were performed three times, at the start of the treatment program, at the end of the 3rd week and 6th week. A patient assessment form was completed at the first assessment session. Data including age, height, weight, and time after surgery were collected.

Pain was assessed before activity using the visual analog scale (VAS) (16), comprising a $100 \mathrm{~mm}$ line with 0 representing 'no pain' and 10 representing 'the worst pain' (17).

The 'Lower Extremity Functional Score' (LEFS), a self-reported questionnaire scoring system, was used to assess knee function (18). This questionnaire consists of 20 items ranging from $0-4$ points. Scores are reported on a $0-$ to $80-$ point scale, with higher scores correlating with better functionality.
The center of gravity (COG) and balance were assessed with the Nintendo $\mathrm{Wii}^{\odot}$ balance board. The Nintendo $\mathrm{Wii}^{\odot}$ balance board is a part of the Nintendo $\mathrm{Wii}^{\odot}$ game console that measures the balance and COG with pressure sensors. It can be used for evaluation and training purposes (19). This research was carried out in accordance with the Helsinki Declaration.

\subsection{Statistical analysis}

Statistical analysis were performed using SPSS v.11.5 for Windows software. Conformity of the data to normal distribution was analysed with the Shapiro-Wilks test. Comparison of treatment groups was performed using an intention-to-treat analysis of difference between baseline-final scores with $95 \%$ confidence intervals. The Wilcoxon Signed-Rank test was used to compare outcomes before and after treatment. The Mann-Whitney U test was used to compare improvements and differences between groups. A value of $p<0.05$ was considered statistically significant for all analyses for a two tailed test.

\section{RESULTS}

A total of 51 patients who have undergone arthroscopic $A C L$ reconstruction were screened for eligibility. Thirty patients (59 $\%)$ participated in the study (Figure 1). After the randomisation process, the patient distributions were as follows: the Nintendo group ( $n=15)$, the control group $(n=15)$. A total of 22 patients were able to complete the study and assessments.
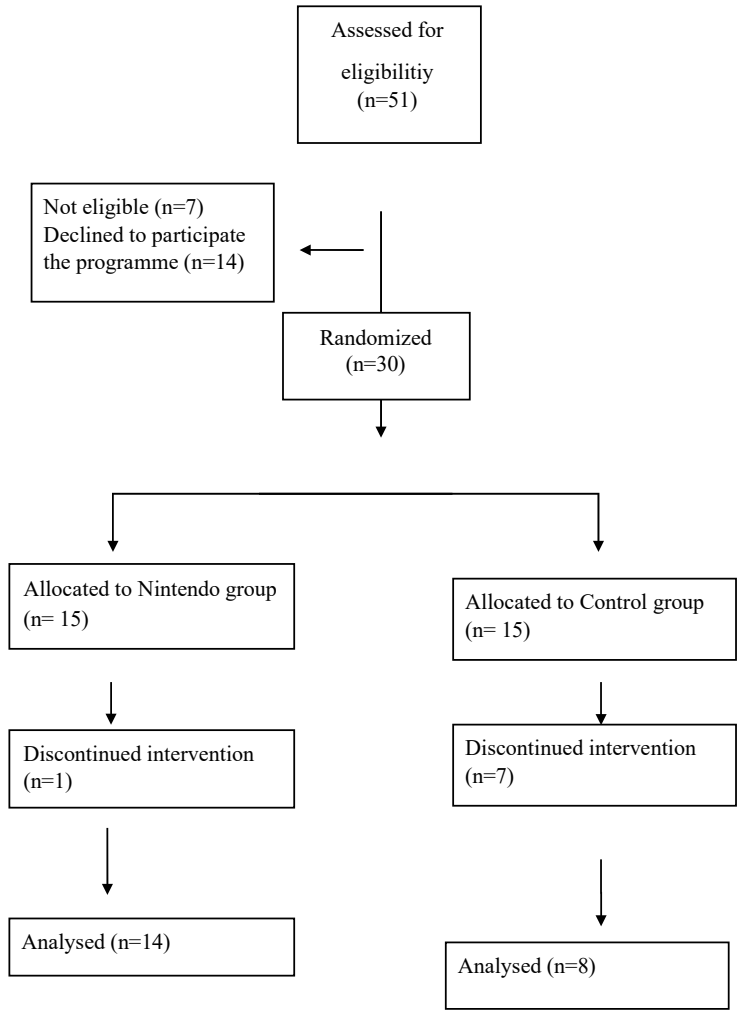

Figure 1. Flow diagram of the participants. 
There were no significant differences between participants in the two groups for any demographic characteristics and baseline assessment scores and (Table 1 and 2). There were no significant differences between the groups in respect of time since surgical reconstruction $(p=0.945)$. The baseline, $3^{\text {rd }}$ and $6^{\text {th }}$ week pain levels did not differ between the groups $(p=0.365,0.676$ and 0.639$)$. At the end of the treatment program, the pain values were significantly reduced in the Nintendo group ( $p=0.005)$, but there was no statistically significant change in the control group $(p=0.058)$. In a comparison of the groups in terms of the pain score changes, there were no significant differences between the two groups (Table 3).

Table 1. Comparison of Baseline Demographics and Surgery Time in Groups

\begin{tabular}{|c|c|c|c|}
\hline & $\begin{array}{l}\text { Nintendo Group } \\
(\mathrm{n}=14) \\
\text { Mean } \pm \mathrm{SD}^{\mathrm{a}}\end{array}$ & $\begin{array}{l}\text { Control Group } \\
(n=8) \\
\text { Mean } \pm S D^{a}\end{array}$ & $\mathbf{p}^{\mathrm{b}}$ \\
\hline Age (year) & $31 \pm 8.41$ & $24 \pm 5.94$ & 0.123 \\
\hline Height $(\mathrm{cm})$ & $174.5 \pm 5.80$ & $178 \pm 7.30$ & 0.245 \\
\hline Weight (kg) & $78 \pm 10.41$ & $76.50 \pm 15.34$ & 0.393 \\
\hline $\mathrm{BMI}^{\mathrm{c}}\left(\mathrm{kg} / \mathrm{m}^{2}\right)$ & $26.21 \pm 2.72$ & $22.80 \pm 4.81$ & 0.101 \\
\hline $\begin{array}{l}\text { Time passed after } \\
\text { surgery (days) }\end{array}$ & $\begin{array}{l}38.5 \pm 40.5 \\
24(13-170)\end{array}$ & $\begin{array}{l}26.6 \pm 14.09 \\
21(18-60)\end{array}$ & 0.945 \\
\hline
\end{tabular}

a Standart Deviation

${ }^{b}$ Significance value in comparison between groups by Mann Whitney U Test ${ }^{c} B M I:$ Body mass index

Table 2. Measurement Results at Baseline, Week 3 and 6

\begin{tabular}{|c|c|c|c|c|}
\hline \multicolumn{2}{|c|}{ Measurements } & \multirow{2}{*}{$\begin{array}{l}\text { Nintendo Group } \\
(n=14) \\
\text { Mean } \pm S D^{\mathrm{a}} \\
3.9 \pm 1.1\end{array}$} & \multirow{2}{*}{$\begin{array}{l}\text { Control Group } \\
(n=8) \\
\text { Mean } \pm \text { SD }^{\mathrm{a}} \\
3.1 \pm 1.8\end{array}$} & \multirow{2}{*}{$\begin{array}{l}p^{b} \\
0.365\end{array}$} \\
\hline \multirow{3}{*}{ Pain } & Baseline & & & \\
\hline & 3rd week & $2 \pm 1.79$ & $2.5 \pm 1.48$ & 0.676 \\
\hline & 6th week & $1 \pm 1.07$ & $1.5 \pm 1.16$ & 0.639 \\
\hline \multirow{3}{*}{ LEFS } & Baseline & $31.5 \pm 16.25$ & $40.5 \pm 13.10$ & 0.452 \\
\hline & 3rd week & $51 \pm 11.68$ & $59.5 \pm 9.25$ & 0.585 \\
\hline & 6th week & $69 \pm 9.47$ & $70.5 \pm 4.40$ & 0.758 \\
\hline \multirow{3}{*}{$\begin{array}{l}\text { COG- } \\
\text { effected } \\
\text { side (\%) }\end{array}$} & Baseline & $41.25 \pm 6.94$ & $40 \pm 11.53$ & 0.864 \\
\hline & 3rd week & $46.40 \pm 4.53$ & $49.50 \pm 5.24$ & 0.055 \\
\hline & 6th week & $49 \pm 4.70$ & $50.20 \pm 5.11$ & 0.561 \\
\hline \multirow{3}{*}{$\begin{array}{l}\text { Balance } \\
\text { score } \\
\text { (sec) }\end{array}$} & Baseline & $11 \pm 8.06$ & $15 \pm 6.84$ & 0.372 \\
\hline & 3rd week & $17 \pm 7.74$ & $16.5 \pm 7.40$ & 0.754 \\
\hline & 6th week & $24 \pm 6.59$ & $21 \pm 7.19$ & 0.593 \\
\hline
\end{tabular}

${ }^{a}$ Standart Deviation

${ }^{b}$ Significance value in comparison between groups by Mann Whitney U Test

The baseline, $3^{\text {rd }}$ and $6^{\text {th }}$ week LEFS levels did not differ between the groups ( $p=0.452,0.585$ and 0.758$)$. In consecutive assessment sessions, the LEFS scores of both groups improved statistically ( $p=0.002$ and 0.012 ), although neither group showed any superiority to the other in terms of LEFS changes ( $p=0.811,0.516$ ve 0.393 ; Table 3 ).

In the baseline assessment, the limbs were asymmetrically loaded in both groups and COG was displaced to the unaffected extremity $(p=0.002$ and 0.024$)$. The COG asymmetry improved statistically significantly in the Nintendo group $(p=0.006)$, after 6 weeks of rehabilitation, but the change in the control group was not statistically significant $(p=0.106)$. The changes in the COG positioning were compared between the two groups, and there were no statistically significant differences in any of the assessment results ( $p=0.277,0.717,0.707$ ) (Table 3 ). However, at the end of the treatment, the location of the COG was statistically symmetrical in both groups ( $p=0.345$ and 0.944$)$.

The baseline, $3^{\text {rd }}$ and $6^{\text {th }}$ week balance levels did not differ between the groups ( $p=0.372,0.754$ and 0.593$)$. When the intra-group changes were analysed at the end of the 6 week treatment program, the balance score changes in both groups were not significant ( $p=0.075$ ve 0.161 ). According to the measurements, statistically significant differences were not observed in the comparison of the balance score changes between the groups in different assessment sessions (Table $3)$.

Table 3. Mean Change in VAS, Functionality, Center of Gravity and Balance Scores from Baseline to Week 3 and 6, from Week 3 to 6

\begin{tabular}{|c|c|c|c|c|}
\hline \multicolumn{2}{|l|}{ Mean Change } & $\begin{array}{l}\text { Nintendo Group } \\
\text { ( } n=14 \text { ) } \\
\text { Mean } \pm S D^{a}\end{array}$ & $\begin{array}{l}\text { Control Group } \\
(n=8) \\
\text { Mean } \pm S^{a}\end{array}$ & $\mathbf{p}^{b}$ \\
\hline \multirow{3}{*}{ Pain } & $\mathrm{C1}^{\mathrm{c}}$ & $2 \pm 1.96$ & $1 \pm 2.13$ & 0.298 \\
\hline & $\mathrm{C2}^{\mathrm{d}}$ & $3 \pm 1.20$ & $1.5 \pm 1.85$ & 0.397 \\
\hline & $\mathrm{C3}^{\mathrm{e}}$ & $1 \pm 2.38$ & $0.5 \pm 2.35$ & 0.256 \\
\hline \multirow{3}{*}{ LEFS } & $\mathrm{Cl}^{\mathrm{c}}$ & $17.50 \pm 13.79$ & $18 \pm 15.09$ & 0.811 \\
\hline & $\mathrm{C2}^{\mathrm{d}}$ & $12 \pm 11.86$ & $7 \pm 8.02$ & 0.516 \\
\hline & $\mathrm{C3}^{\mathrm{e}}$ & $35.50 \pm 18.77$ & $27.50 \pm 16.65$ & 0.393 \\
\hline \multirow{3}{*}{ COG (\%) } & $\mathrm{C}^{\mathrm{c}}$ & $2 \pm 5.29$ & $5 \pm 12.71$ & 0.277 \\
\hline & $\mathrm{C2}^{\mathrm{d}}$ & $3 \pm 6.03$ & $2.5 \pm 6.92$ & 0.717 \\
\hline & $\mathrm{C3}^{\mathrm{e}}$ & $8.3 \pm 7.18$ & $7.70 \pm 15.56$ & 0.707 \\
\hline \multirow{3}{*}{$\begin{array}{l}\text { Balance score } \\
\text { (sec) }\end{array}$} & $\mathrm{C}^{\mathrm{c}}$ & $4 \pm 8.27$ & $0 \pm 6.53$ & 0.305 \\
\hline & $\mathrm{C2}^{\mathrm{d}}$ & $1 \pm 5.19$ & $7.5 \pm 12.93$ & 0.246 \\
\hline & $\mathrm{C3}^{\mathrm{e}}$ & $4 \pm 8.30$ & $4 \pm 11.23$ & 1.000 \\
\hline
\end{tabular}

${ }^{a}$ Standart Deviation

${ }^{b}$ The statistical significance of the comparison of the differences between the evaluations

${ }^{c} \mathrm{C} 1$ : Mean change from baseline to week 3 ,

${ }^{d} \mathrm{C} 2$ : Mean change from week 3 to week 6 ,

${ }^{e} \mathrm{C} 3$ : Mean change from baseline to week 6. sec: second, \%: percent

\section{DISCUSSION}

The results of this study indicate that adding the Nintendo $\mathrm{Wii}^{\odot}$ virtual games to the accelerated rehabilitation program does not provide additional benefit in terms of pain, functionality, COG and balance parameters in the early period of $A C L$ reconstruction.

ACL-reconstruction aims to restore joint stability and prevent osteoarthritis and the risk of secondary meniscal tears (20) and an individualized postoperative rehabilitation program is necessary for a stable functional knee. Rehabilitation programs after $A C L$ reconstruction are implemented at different intensities and for different durations (7, 21-23). 
In this study, the individual exercise therapy sessions were performed for 45 minutes, 3 times/week (18 sessions) and the Nintendo $\mathrm{Wii}^{\odot}$ balance games were added to the Nintendo group, for 40 minutes, 3 times/week after the $3^{\text {rd }}$ week of the rehabilitation protocol. A 2-5-year follow-up of 604 patients after arthroscopic ACL reconstruction, reported that moderate to severe subjective anterior knee pain related to activity, walking up and down stairs, and sitting with the knee flexed was found in $33.6 \%$ of patients (24).

In another study, significant knee pain and symptoms were found in $9 \%-39 \%$ of first - time ACL reconstruction patients at 6 years (20). Furthermore, it has been claimed that pain affects functioning (25).

Despite receiving treatment following reconstruction, a decrease in functionality has been reported in approximately $50 \%$ of cases (26). Many factors, such as quadriceps activity, decreased strength, decreased EMG activity of vastus medialis (VM) and vastus lateralis (VL) muscles, and increased musculotendinous stiffness, are considered to affect knee function after $A C L$ reconstruction compared to the unaffected extremity (27).

Post-surgery rehabilitation programs and visual biofeedback exercises are methods that improve functionality (23, 27). A previous study has shown that Nintendo Wii games with visual and auditory biofeedback and traditional physiotherapy approaches have no superiority in terms of functional outcomes in post-op ACL rehabilitation (23). In this study, a significant improvement was observed in the pain values after the treatment program in the Nintendo group received Nintendo $\mathrm{Wii}^{\odot}$ balance games added to the accelerated rehabilitation program. When the intra-group change of functionality scores were analysed, improvements were obtained in the scores of both groups after treatment. However, no superiority of either groups was determined in terms of changes in pain and functional scores. According to the results of the study, Nintendo $\mathrm{Wii}^{\odot}$ balance games do not appear to provide additional advantages to the accelarated rehabilitation program in respect of improving pain and functionality in the early period after the reconstruction.

Changes in weight loading and reduced postural control are seen in individuals who have undergone $A C L$ reconstruction (7). Although it has been suggested in the literature that these changes are seen in the short term (28) it has also been indicated that these could extend to 2 years after reconstruction (29). It has been reported that this condition may be caused by weak sensory input due to changes in mechanoreceptor and central nerve connections (30).

The average location of the weight of an object is the center of gravity (COG). When an individual stands on a Nintendo $\mathrm{Wii}^{\odot}$ balance board, the COG of the subject is detected and displayed as feedback on the screen. This can then be used to encourage and improve the motor learning of the subject (31).

At the baseline of this study, COG asymmetry was present in both groups and symmetric COG distribution was obtained in the two groups after the 6 - week rehabilitation program. In one case study, COG symmetry was obtained in the $3^{\text {rd }}$ week of Nintendo $\mathrm{Wii}^{\mathrm{O}}$ balance board exercises after posterior cruciate ligament reconstruction (22) but no comparison was possible because no other treatment approach was applied in this study.

When intra-group change was analyzed in this study before and after treatment, the mean COG distribution change was statistically significant in the Nintendo group. However, when the mean change amounts in COG distribution were compared, it was seen that the groups were similar and adding 3 weeks of Nintendo $\mathrm{Wii}^{\odot}$ balance board exercises was not superior to the accelerated rehabilitation program applied alone. Due to the limited follow-up period of the current study patient population, it was not possible to determine the effect of Nintendo $\mathrm{Wii}^{\odot}$ balance games on COG and other parameters in the long term.

Changes in neuromuscular control occur with damage to the mechanoreceptors after ACL injury (32). Continuation of damage to the mechanoreceptors (7), and poor proprioception lead to a deterioration in balance $(4,16)$, even if the mechanical stability of the knee is provided by reconstruction.

The results of this study showed that adding Wii games to the rehabilitation protocol did not provide superiority to the change of balance scores. Regaining dynamic joint stability between the 20th and the 32nd weeks after surgery may be seen as a reason for the failure of balance improvement in the early stage. It has also been noted in literature that earlystage balance training in $A C L$ reconstructed individuals was not effective on balance (10).

A stable Nintendo $\mathrm{Wii}^{\circ}$ balance board, providing an easy assessment for young and physically active patients, could have been another reason that similar balance score changes were obtained in both groups. Dynamic and more difficult balance assessments may provide more sensitive information (30).

A previous study reported that a reconstructed group had significantly lower balance scores than a healthy group in balance assessment, which included standing on one foot, but no difference in assessment when standing on both feet (33). Another important issue is that the eyes have to be open in the standing balance assessment with the Nintendo $\mathrm{Wii}^{\odot}$ balance board. The balance system is complex and, vestibular, visual, and somatosensory/proprioceptive sensory systems, provide our sense of balance. That worse balance scores have been reported in balance assessments performed with the eyes closed may indicate the visual system compensation mechanism (34). It can be considered that no differences were determined between the groups in respect of balance improvements because of the contribution of visual feedback and that the assessment was made using a static balance board.

Studies in literature, examining the effects of virtual rehabilitation and Nintendo $\mathrm{Wii}^{\odot}$ on balance and lower 
extremity functioning in individuals with $A C L$ reconstruction are rather limited. This study is one of the few studies in this area. The limited time of the study, not including longterm follow up, small sample size, and assessment balance scores with static and simple measurement can be accepted as limitations of this study. The results of this study need to be confirmed on larger samples with a randomized control group with long term follow-up.

\section{CONCLUSION}

The results of this study showed that the addition of Nintendo $\mathrm{Wii}^{\odot}$ balance games played with the Nintendo Wii Balance Board in the last 3 weeks of the 6 - week rehabilitation program did not provide additional benefits to the accelerated $A C L$ rehabilitation protocol in respect of the parameters assessed. However, the clinical observations showed that patients could become bored with the repetitive exercises of the rehabilitation program and had more enjoyment with the Nintendo $\mathrm{Wii}^{\circ}$ balance games, which are becoming widespread with the development of technology. Patients reported that they played the games with active participation, found them enjoyable but not did not become exhausted. It can be said that the Nintendo $\mathrm{Wii}^{\odot}$ games are easy to use and inexpensive. Nintendo $\mathrm{Wii}^{\odot}$ balance board exercises can be more suitable for improving balance and symmetrical weight-bearing in the rehabilitation of sedentary and geriatric populations with neurological deficit.

Financial Disclosure and Conflicts of Interest: The authors declare that they have no conflicts of interest. This study funded by Marmara University, Scientific Research Project Coordination Unit (Project No: SAG-C-YLP-130.116.0004).

\section{REFERENCES}

[1] Spindler KP, Wright RW. Anterior cruciate ligament tear. New Engl J Med 2008; 359 (20): 2135 - 2142.

[2] Riemann BL, Lephart SM. The sensorimotor system, part II: the role of proprioception in motor control and functional joint stability. J Athl Training 2002; 51 (4): $80-84$.

[3] Vathrakokilis K, Malliou P, Giofsidou A, Beneka A, Godolias $G$. Effects of a balance training protocol on knee joint proprioception after anterior cruciate ligament reconstruction. J Back Musculoskelet 2008; 21 (4): 233 - 237.

[4] Gokeler A, Benjaminse A, Hewett TE, Lephart SM, Engebretsen L, Ageberg E, Engelhardt M, Arnold MP, Postema K, Otten E, Dijkstra PU. Proprioceptive deficits after ACL injury: are they clinically relevant? Brit J Sport Med 2012; 46 (3): 180 - 192.

[5] Clark RA, Howells B, Pua Y-H, Feller J, Whitehead T, Webster KE. Assessment of standing balance deficits in people who have undergone anterior cruciate ligament reconstruction using traditional and modern analysis methods. J Biomech 2014; 47 (5): $1134-1137$.

[6] Schroeder MJ, Krishnan C, Dhaher YY. The influence of task complexity on knee joint kinetics following $\mathrm{ACL}$ reconstruction. Clin Biomech 2015; 30 (8): 852 - 859.

[7] Howells BE, Clark RA, Ardern CL, Bryant AL, Feller JA, Whitehead TS, Webster KE. The assessment of postural control and the influence of a secondary task in people with anterior cruciate ligament reconstructed knees using a Nintendo Wii Balance Board. Brit J Sport Med 2013; 47 (14): 914 - 919.

[8] Mikkelsen C, Werner S, Eriksson E. Closed kinetic chain alone compared to combined open and closed kinetic chain exercises for quadriceps strengthening after anterior cruciate ligament reconstruction with respect to return to sports: a prospective matched follow - up study. Knee Surg Sport Tr A 2000; 8 (6): $337-342$.

[9] Van Grinsven S, Van Cingel REH, Holla CJM, Van Loon CJM. Evidence-based rehabilitation following anterior cruciate ligament reconstruction. Knee Surg Sport Tr A 2010; 18 (8): $1128-1144$.

[10] Cooper R, Taylor N, Feller JA. Systematic review of the effect of proprioceptive and balance exercises on people with an injured or reconstructed anterior cruciate ligament. Res Sports Med 2005; 13 (2): 163 - 178.

[11] Graves LE, Ridgers ND, Stratton G. The contribution of upper limb and total body movement to adolescents' energy expenditure whilst playing Nintendo Wii. Eur J Appl Physiol 2008; 104 (4): 617.

[12] Laver K, Ratcliffe J, George S, Burgess L, Crotty M. Is the Nintendo Wii Fit really acceptable to older people?: a discrete choice experiment. BMC Geriatr 2011; 11 (1): 64.

[13] Bryanton C, Bosse J, Brien M, Mclean J, Mccormick A, Sveistrup $\mathrm{H}$. Feasibility, motivation, and selective motor control: virtual reality compared to conventional home exercise in children with cerebral palsy. Cyberpsychol Behav 2006; 9 (2): $123-128$.

[14] Available from: https://www.randomizer.org/. Accessed Date: 10.01.2016

[15] Shelbourne KD, Nitz P. Accelerated rehabilitation after anterior cruciate ligament reconstruction. Am J Sport Med 1990; 18 (3): $292-299$.

[16] Kouvelioti V, Kellis E, Kofotolis N, Amiridis I. Reliability of singleleg and double-leg balance tests in subjects with anterior cruciate ligament reconstruction and controls. Res Sports Med 2015; 23 (2): 151 - 166.

[17] Bijur PE, Silver W, Gallagher EJ. Reliability of the visual analog scale for measurement of acute pain. Acad Emerg Med 2001; 8 (12): $1153-1157$.

[18] Binkley JM, Stratford PW, Lott SA, Riddle DL. The lower extremity functional scale (LEFS): scale development, measurement properties, and clinical application. Phys Ther 1999; 79 (4): 371 - 83.

[19] Pigford T, Andrews WA. Feasibility and benefit of using the Nintendo Wii Fit for balance rehabilitation in an elderly patient experiencing recurrent falls. J Stud Phys Ther Res 2010: 2.

[20] Wasserstein D, Huston LJ, Nwosu S, MOON Group, Spindler KP. KOOS pain as a marker for significant knee pain two and six years after primary $\mathrm{ACL}$ reconstruction: a Multicenter Orthopaedic Outcomes Network (MOON) prospective longitudinal cohort study. Osteoarthr Cartilage 2015; 23 (10): $1674-1684$.

[21] Mikkelsen C, Werner S, Eriksson E. Closed kinetic chain alone compared to combined open and closed kinetic chain exercises for quadriceps strengthening after anterior cruciate ligament reconstruction with respect to return to sports: a prospective matched follow-up study. Knee Surg Sport $\operatorname{Tr}$ A 2000; 8 (6): 337 -342 . 
[22] Puh U, Majcen N, Hlebs S, Rugelj D. Effects of Wii balance board exercises on balance after posterior cruciate ligament reconstruction. Knee Surg Sport Tr A 2014; 22 (5): 1124- 1130.

[23] Baltacı G, Harput G, Haksever B, Ulusoy B, Özer H. Comparison between Nintendo Wii Fit and conventional rehabilitation on functional performance outcomes after hamstring anterior cruciate ligament reconstruction: prospective, randomized, controlled, double-blind clinical trial. Knee Surg Sport Tr A 2013; 21 (4): $880-887$.

[24] Kartus J, Magnusson L, Stener S, Brandsson S, Eriksson BI, Karlsson J. Complications following arthroscopic anterior cruciate ligament reconstruction A 2-5-year follow-up of 604 patients with special emphasis on anterior knee pain. Knee Surg Sport Tr A 1999; 7 (1): 2 - 8.

[25] Gülbahar S, Akgün B, Karasel S, Baydar M, El O, Pınar H. The effect of anterior knee pain on strength, functional tests, proprioception and balance after anterior cruciate ligament reconstruction. Türk Fiz Tip Rehab D 2013; 59 (2): 90 - 97.

[26] Acevedo R, Rivera-Vega A, Miranda G, Micheo W. Anterior cruciate ligament injury: identification of risk factors and prevention strategies. Curr Sport Med Rep 2014; 13 (3): 186 $-191$.

[27] Bryant AL, Kelly J, Hohmann E. Neuromuscular adaptations and correlates of knee functionality following $\mathrm{ACL}$ reconstruction. J Orthop Res 2008; 26 (1): 126 - 135.

[28] Clark RA, McGough R, Paterson K. Reliability of an inexpensive and portable dynamic weight bearing asymmetry assessment system incorporating dual Nintendo Wii Balance Boards. Gait Posture 2011; 34 (2): 288 - 291.

[29] Paterno MV, Schmitt LC, Ford KR, Rauh MJ, Hewett TE. Biomechanical measures during landing and postural stability predict second anterior cruciate ligament injury after anterior cruciate ligament reconstruction and return to sport. Am J Sport Med 2010; 38 (10): 1968 - 1978.

[30] Brunetti O, Filippi GM, Lorenzini M, Liti A, Panichi R, Roscini M, Pettorossi VE, Cerulli G. Improvement of posture stability by vibratory stimulation following anterior cruciate ligament reconstruction. Knee Surg Sport Tr A 2006; 14 (11): 1180 1187.

[31] Swanson LR, Lee TD. Effects of aging and schedules of knowledge of results on motor learning. J Gerontol 1992; 47 (6): $406-411$.

[32] Ma Y, Deie M, Iwaki D, Asaeda M, Fujita N, Adachi N, Ochi Mitsuo. Balance ability and proprioception after singlebundle, single-bundle augmentation, and double - bundle ACL reconstruction. Sci World J 2014; 2014:1-8.

[33] Smith MD, Bell DR. Negative effects on postural control after anterior cruciate ligament reconstruction as measured by the balance error scoring system. J Sport Rehabil 2013; 22 (3): 224 $-228$.

[34] Molka AZ, Lisiński P, Huber J. Visual biofeedback exercises for improving body balance control after anterior cruciate ligament reconstruction. J Phys Ther Sci 2015; 27 (7): 2357 2360 .

How to cite this article: Karakoc Z. B., Kuru Colak T., Sari Z., Polat M.G. The Effect of Virtual Rehabilitation Added to an Accelerated Rehabilitation Program After Anterior Cruciate Ligament Reconstruction: A Randomized Controlled Trial. Clin Exp Health Sci 2019; 9: 124-129. DOI: $10.33808 /$ clinexphealthsci.564273 